\title{
Rossby wave instability and high-frequency quasi-periodic oscillations in accretion discs orbiting around black holes
}

\author{
P. Varniere ${ }^{1,2}$, F. Casse ${ }^{1,2}$, and F. H. Vincent ${ }^{3}$ \\ 1 Laboratoire AstroParticule et Cosmologie, Université Paris Diderot, CNRS/IN2P3, CEA/Irfu, Observatoire de Paris, \\ Sorbonne Paris Cité, 10 rue Alice Domon et Léonie Duquet, 75205 Paris Cedex 13, France \\ e-mail: varniere@apc . univ-paris7.fr \\ 2 Laboratoire AIM, CEA/IRFU-CNRS/INSU-Université Paris Diderot, CEA DRF/IRFU/DAp, 91191 Gif-sur-Yvette, France \\ 3 LESIA, Observatoire de Paris, Université PSL, Sorbonne Université, Université Paris Diderot, Sorbonne Paris Cité, \\ 5 place Jules Janssen, 92195 Meudon, France
}

Received 5 February 2019 / Accepted 12 April 2019

\begin{abstract}
Context. The rather elusive high-frequency quasi-periodic oscillations (HFQPOs) observed in the X-ray light curve of black holes have been seen in a wide range of frequencies, even within one source. Also notable is the detection of "pairs" of HFQPOs with a close-to-integer ratio between the frequencies.

Aims. The aim of this paper is to investigate some of the possible observables that we could obtain from the Rossby wave instability (RWI) active in the accretion disc surrounding the compact object.

Methods. Using the newly developed GR-AMRVAC code able to follow the evolution of the RWI in a full general relativistic framework, we explore how RWI can reproduce observed HFQPO frequency ratios and whether or not it is compatible with observations. In order to model the emission coming from the disc we have linked our general relativistic simulations to the general relativistic ray-tracing GYOTO code and delivered synthetic observables that can be confronted with actual data from binary systems hosting HFQPOs.

Results. We demonstrate that some changes in the physical conditions prevailing in the part of the disc where RWI can be triggered lead to various dominant RWI modes whose ratio recovers frequency ratios observed in various X-ray binary systems. In addition we also show that when RWI is triggered near to the last stable orbit of a spinning black hole, the amplitude of the X-ray modulation increases with the spin of the black hole. Revisiting published data on X-ray binary systems, we show that this type of relationship actually exists in five systems where an indirect measurement of the spin of the black hole is available.
\end{abstract}

Key words. stars: black holes - X-rays: binaries

\section{High-frequency quasi-periodic oscillations in $\mathrm{X}$-ray binary systems}

While fast variability is often detected in black hole binaries, the most sought after observations are those of high-frequency quasi-periodic oscillations (HFQPOs). These HFQPOs appear as narrow peak(s) in the X-ray powerdensity spectra (PDSs) of black hole binaries and have been detected in eight different black hole sources (GRO J1655-40, GRS 1915+105, XTE J1550-564, H1743-322, 4U 1630-47, XTE J1650-500, XTE J1859+226 IGR J17091-3624; e.g. Remillard \& McClintock 2006; Altamirano \& Belloni 2012; Belloni et al. 2012, and references therein) ranging from as low as $27 \mathrm{~Hz}$ in GRS $1915+105$ (Belloni et al. 2001) up to a few hundred Hertz (a maximum of $450 \mathrm{~Hz}$ has been detected in GRO J1655-40 Remillard \& McClintock 2006). High-frequency quasi-periodic oscillations are particularly interesting as their frequencies typically lie in the frequency range of the Keplerian frequency of the last stable orbit around the central black hole. They can therefore be seen as a window to the innermost region of accretion where strong gravity is expected to play an important role ${ }^{1}$. This is one of the reasons why HFQPOs,

\footnotetext{
1 Relativistic effects and their influences on the properties of LFQPOs are for example discussed within the context of the accretion-ejection instability in Varnière et al. (2012).
}

although much weaker and rarer, have stimulated much more interest than their low-frequency counterparts (LFQPO).

Another reason behind the interest in HFQPOs is that they sometimes exhibit an integer ratio between detected peaks, such as the classical 1:2 in GRS 1915+105 Belloni \& Altamirano (2013), but also a 2:3 ratio in, for example, GRO J1655-40 (Remillard et al.2002) and both the 2:3 and potentially 3:4 ratios in XTEJ1550-564 (Miller et al. 2001; Varnière \& Rodriguez 2018). Those occurrences lead to a wide variety of models. Here we focus on one of them based on the Rossby wave instability (RWI).

The RWI, while first introduced in the context of galactic discs by Lovelace \& Hohlfeld (1978), was rarely used because of the difficulty in finding a physical setup fulfilling its criterion. It is only in the past decade that the RWI has been more widely used, as physical settings where it could be triggered were discovered from planet formation (Varnière \& Tagger 2006; Meheut et al. 2010; Lyra \& Mac Low 2012; Lin 2012) to the flares of Sgr A* (Tagger \& Melia 2006; Vincent et al. 2014) and the fast variability of microquasars (Tagger \& Varnière 2006; Varnière et al. 2011, 2012; Vincent et al. 2013). This last case comes from the fact that the epicyclic frequency goes to zero at the last stable orbit, therefore ensuring that the criterion for the RWI is fulfilled inside the inner region of the disc.

This leads us to study in a full general relativistic framework the existence and evolution of the RWI in the case of a 
Schwarzschild (Casse et al. 2017) and Kerr (Casse \& Varniere 2018) black hole. Here we take this a step further by adding general relativistic ray-tracing to our simulation in order to create numerical observations that can be translated into observables such as the root mean square (rms) amplitude of the modulation created by the instability, its quality factor, and the presence of integer ratio peaks in the PDS.

\section{The Rossby wave instability as a model for HFQPOs}

Here we review the salient points that make the RWI a good candidate to explain the HFQPOs observed in microquasars. We refer to Lovelace \& Romanova (2014) for a full review of the RWI in its different domain of application.

- Thanks to the vanishing of the epicyclical frequency at the last stable orbit, this hydrodynamical instability occurs naturally in any disc that is getting close to its last stable orbit (Tagger \& Varnière 2006).

- Furthermore, when fully developed, the RWI may exhibit several unstable modes, each one characterised by a different toroidal integer number $m$. The strongest, dominant mode of the RWI is not always the $m=1$ but higher order modes can be present, simultaneously or not, offering a natural explanation for the different integer ratios observed (Tagger \& Varnière 2006). This stems from the fact that the frequency induced by a RWI mode is directly proportional to the value of $m$.

- Also, the RWI was shown to be able to occur simultaneously with one of the instabilities proposed to be at the origin of the LFQPOs (Varnière et al. 2011, 2012). This is important as HFQPOs and LFQPOs are often observed together. Not many HFQPO models have demonstrated their ability to exist in a disc exhibiting LFQPOs.

- The RWI is one of the few HFQPO models that demonstrates its ability to modulate the X-ray flux up to the observed levels (Vincent et al. 2013).

In order to complete the next step in our exploration of the RWI characteristics and how they relate to the observed HFQPOs, we turn to numerical simulations to obtain more direct information about observables such as the actual PDS peak distribution that rises from the RWI and what it depends on, but also how the rms amplitude changes with the spin of the black-hole.

\section{NOVAs: General relativistic simulations of the Rossby wave instability around spinning black holes}

Casse \& Varniere (2018) performed the first general relativistic hydrodynamical simulation of the RWI in a Kerr metric context proving its existence in the disc around maximally spinning black holes. These authors also concluded that, while there were only limited general relativistic effects on the instability in itself, the effect was mostly related to the time lapse, therefore suggesting a necessity to ray-trace, in a general relativistic context, the emission back to the observer if one wants to look into more details at the impact of the spin. For that reason we build the Numerical Observatory of Violent Accreting systems, NOVAs (Varnière et al. 2018) which couple together the results from our newly developed MPI $^{2}$ code GR-AMRVAC (Casse \& Varniere 2018) with the open-source general relativistic ray-tracing code

2 Message Passing Interface used for parallel computing.
GYOTO (Vincent et al. 2011) to create the light curves and then the PDS associated with our simulations. Using those we are able to track the impact of the spin and the shape of the inner region, where the RWI is active, on the flux modulation.

\subsection{General relativistic hydrodynamical simulations}

The GR-AMRVAC code solves general relativistic hydrodynamics equations including conservation of mass and momentum. Spacetime geometry around a rotating, uncharged black hole is fully determined by the Kerr metric (Kerr 1963). The general expression of any line element $\mathrm{d} s$ in a $(3+1)$ splitting in BoyerLindquist coordinates is

$\mathrm{d} s^{2}=-\alpha^{2}(c \mathrm{~d} t)^{2}+\gamma_{i j}\left(\mathrm{~d} x^{i}+\beta^{i} c \mathrm{~d} t\right)\left(\mathrm{d} x^{j}+\beta^{j} c \mathrm{~d} t\right)$,

where $\alpha$ is the lapse function, $\beta^{i}$ is the shift vector, and $\gamma_{i j}$ is the spatial metric tensor (see e.g. Casse \& Varniere 2018, for definition of these quantities. In our notation, greek letter stand for indices ranging over all coordinates while latin letters are restricted to spatial coordinates only. We use $\gamma$ to represent the determinant of the spatial metric tensor.

Defining $\rho$ as the proper density of the gas and $\mathrm{v}^{i}$ its contravariant Eulerian velocity components (normalized to the speed of light $c$ ) one can express the aforementioned conservation laws as

$$
\begin{aligned}
\partial_{t}(\sqrt{\gamma} D)+\partial_{j}\left(\sqrt{\gamma} D\left(\alpha \mathrm{v}^{j}-\beta^{j}\right)\right)=0 & \\
\partial_{t}\left(\sqrt{\gamma} S_{i}\right)+\partial_{j}\left(\sqrt{\gamma}\left[S_{i}\left(\alpha \mathrm{v}^{j}-\beta^{j}\right)+\alpha P \delta_{i}^{j}\right]\right)= & \sqrt{\gamma}\left\{-\left(W^{2} \rho h c^{2}-P\right) \partial_{i} \alpha\right. \\
& +\frac{\alpha}{2}\left(S^{j} \mathrm{v}^{k}+P \gamma^{j k}\right) \partial_{i} \gamma_{j k} \\
& \left.+S_{j} \partial_{i} \beta^{j}\right\}
\end{aligned}
$$

where $W=\left(1-\mathrm{v}_{i} \mathrm{v}^{i}\right)^{-1 / 2}$ is the Lorentz factor of the gas, $D=W \rho$ is the relativistic mass density, $W^{2} p h c^{2}$ is the relativistic enthalpy of the fluid, and $P$ is the thermal pressure. One can easily define the relativistic momentum of the fluid as $S_{i}=W^{2} \rho h c^{2} \mathrm{v}_{i}$. The full energy conservation includes heating and cooling mechanisms which would require the consideration of physics beyond hydrodynamics, such as high-energy kinetic theory and radiative transfer. Instead, we choose to replace this equation by a much simpler power-law pressure prescription $P=C_{\mathrm{o}} \rho^{\tilde{\gamma}}$ where $C_{\mathrm{o}}$ and $\tilde{\gamma}$ are two positive constants. In order to close the system, we use the standard relativistic equation of state described by Mignone \& McKinney (2007).

We use a similar setup as in Casse \& Varniere (2018) for our simulations. These initial conditions are designed for vertically integrated accretion disc configurations. We adopt such an approach since accretion discs in X-ray binaries are likely to be very thin. We have already shown in Casse et al. (2017) that full 3D simulations of thin accretion discs prone to RWI lead to the same results as vertically integrated simulations in the case of a non-spinning black hole. The vertical gravitational force generated by Kerr black holes exhibits a similar behaviour to that generated around Schwarzschild black holes when close to the equatorial plane. We can therefore assume that the relevance of vertically integrated simulations is still valid for simulations dealing with very thin discs orbiting around Kerr black holes. This assumption is also sustained by the fact that RWI vortices are essentially 2D structures when this instability is triggered in a carefully crafted setup where no other instability is present (Meheut et al. 2012).

Following the criterion of the development of RWI, the unstable zone will then be the region where we have an 

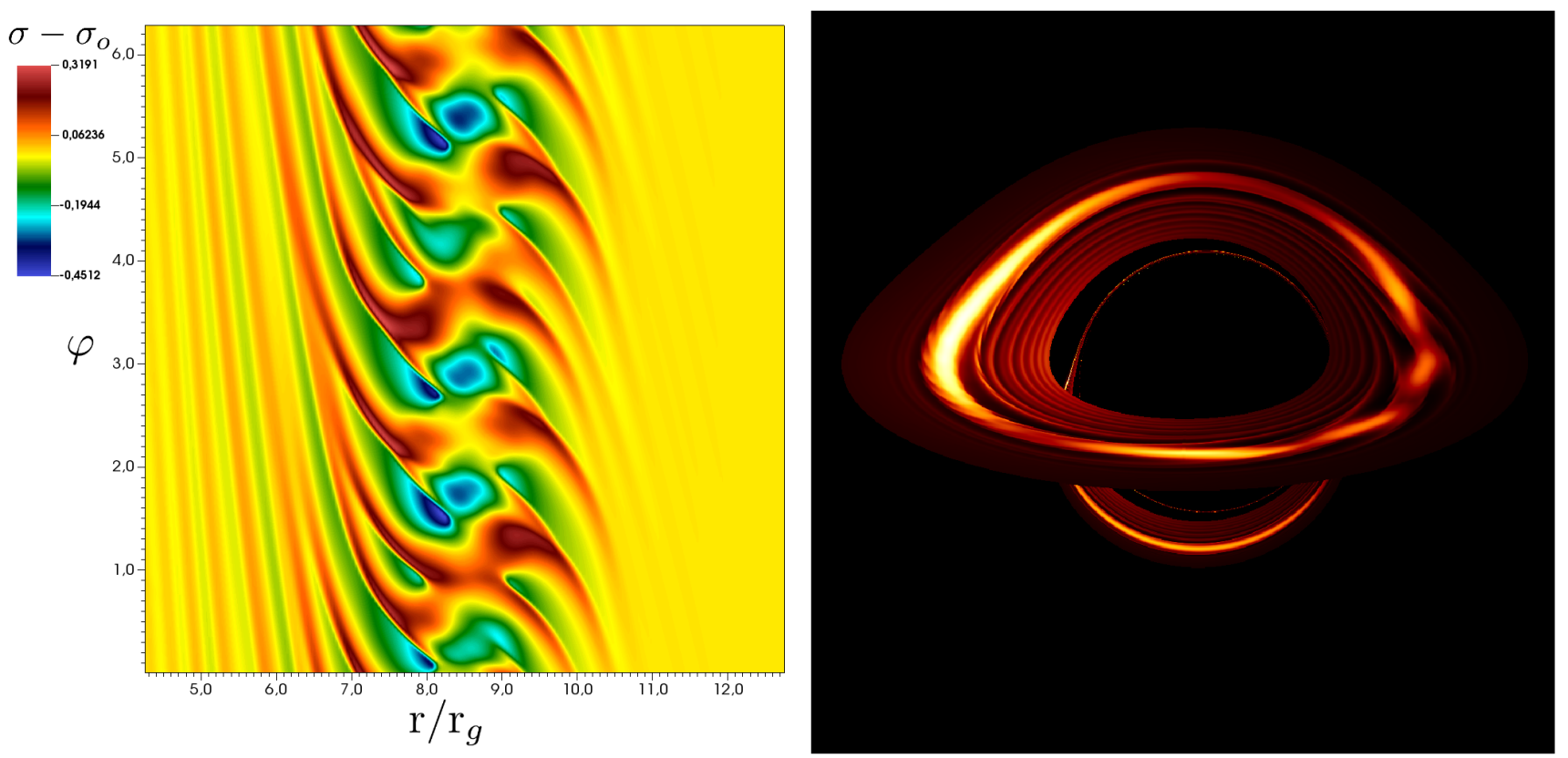

Fig. 1. Left: typical example of RWI triggered in an accretion disc at $r_{\mathrm{b}}=8.5 r_{g}$ around a black hole whose spin parameter is set to 0.5 . Density perturbations are displayed exhibiting the occurrence of vortices located at $r_{\mathrm{b}}$ from the black hole at a late stage of the simulation. Identified vortices are all anti-cyclonic according to the rotation of the disc as anticipated when one considers the RWI. Right: synthetic image from the simulation displayed on the left panel. The GRHD simulation is performed by the GR-AMRVAC code while the image is processed using the GYOTO general relativistic ray-tracing code. This image considers photons (with energy of $2 \mathrm{keV}$ ) reaching a remote observer standing far from the black hole.

extremum of the so-called vortensity $\left(\mathcal{L}=\varepsilon_{j}^{\theta i} \mathrm{v}_{; i}^{j} / \sigma(r), \varepsilon_{\mathrm{k}}^{i j}\right.$ being a Levi-Civita tensor).

The initial conditions defining our simulations consider a surface density $\sigma(r)$ of the disc exhibiting a bump centered around the so-called corotation radius $r_{\mathrm{c}}$. The surface density of the disc is set in a similar fashion as in Casse \& Varniere (2018), namely

$\sigma(r)=\left(\frac{r_{\mathrm{o}}}{r}\right)^{0.7}\left(1+\varepsilon \exp \left\{-\left(\frac{\left(r-r_{\mathrm{c}}\right)^{2}}{2 \delta^{2}}\right)\right\}\right)$,

where $r_{\mathrm{o}}$ corresponds to the last stable orbit radius for the fastest spinning black hole considered $(a=0.995), r_{\mathrm{o}}=1.34 r_{g}$. We ran a much smaller set of spins and positions, while changing the parameters of the unstable zone ${ }^{3} \varepsilon$ and $\delta$. With the profile chosen for those simulations we can succinctly parametrize the unstable zone by the amplitude of the density bump $(\varepsilon)$ and the radial $(\delta)$ extent of the fulfilled RWI criteria.

The radial equilibrium of the disc is achieved when the centrifugal force balances both the gravity of the black hole and the thermal pressure of the gas. This leads to the prescription of the rotational velocity as

$\mathbf{v}^{\varphi}=\frac{-\partial_{\mathrm{r}} \beta^{\varphi}+\sqrt{\left(\partial_{\mathrm{r}} \beta^{\varphi}\right)^{2}+2 \alpha \partial_{\mathrm{r}} \gamma_{\varphi \varphi}\left(\partial_{\mathrm{r}} \alpha+\alpha \frac{\partial_{\mathrm{r}} P}{\rho h W^{2}}\right)}}{\alpha \gamma^{\varphi \varphi} \partial_{\mathrm{r}} \gamma_{\varphi \varphi}}$.

The radial pressure profile $P$ is set following the aforementioned power-law prescription where the parameters $C_{\mathrm{o}}=1.8 \times 10^{-4}$ and $\tilde{\gamma}=3$ as in Casse et al. (2017), Casse \& Varniere (2018).

\footnotetext{
In Casse \& Varniere (2018) the unstable zone was kept unchanged to study only the impact of the spin. We refer to this study to see the full impact of the spin and GR effects on the instability. Here we focus on observables.
}

The values have been chosen in order to be consistent with equatorial conditions consistent with a thin disc whose aspect ratio is $H / r \sim 4 \times 10^{-2}$. Let us note that in our vertically integrated disc context, the disc scale height $H$ is evaluated using the assumption of a vertical hydrostatic equilibrium where the vertical pressure gradient is expected to balance to vertical gravity.

The instability is then triggered by injecting very-smallamplitude random velocity fluctuations near the corotation radius. In all the simulations, the disc goes from its last stable orbit to about three times that, while we manually centered the unstable zone by choosing the aforementioned density profile. The grid resolution is $196 \times 600$ for each simulation while the spatial domain ranges from $r_{\text {LSO }}$ to $3 r_{\text {LSO }}$ and $\phi \in[0,2 \pi]$. The radial boundaries are continuous while azimuthal boundaries are periodic. In order to time advance, we used a Harten, Lax, and van Leer (HLL) solver linked to a Koren slope limiter. A typical simulation requires approximately several tens of thousands of time steps and lasts a few hundred cpu-hours.

The left figure of Fig. 1 shows the density profile for the case of $a=0.5$ with the RWI triggered at $r_{\mathrm{c}}=8.5 r_{g}$. As we are using a subset of our simulations presented in Casse \& Varniere (2018), we refer the reader interested in a more in-depth study of the RWI behaviour to that paper. Here we focus more on the possible observational consequences of the GR effects.

\subsection{General relativistic ray-tracing to the observer}

For all ray-tracing computations in this article, we use the opensource $^{4}$ GYOTO code. Photons are traced backwards in time by integrating the geodesic equation using a Runge-Kutta-Fehlberg adaptive-step integrator at order 7/8 (meaning that the method

\footnotetext{
4 Freely available at http: //gyoto.obspm. fr.
} 
is eighth order, with an error estimation at seventh order). From such maps of specific intensity, the light curve (flux as a function of time) is derived by summing all pixels weighted by the element of solid angle, which is subtended by each pixel. GYOTO computations consider the very same Kerr metric as the hydrodynamical simulations, so that all general relativistic effects upon photons are taken into account in our study, namely gravitational frequency shift and time lapse.

A backwards-integrated photon is traced until the accretion disc is reached. The local temperature is derived from the 2D grid of the GRHD simulation by considering the relation $T(\rho)=T_{\text {inner }}\left(\sigma / \sigma_{\text {inner }}\right)^{\gamma_{\mathrm{o}}-1}$ where $\sigma$ is the local density interpolated from the grid, $\sigma_{\text {inner }}$ is the density at the inner radius of the disc, $T_{\text {inner }}$ is the temperature at the inner radius, fixed at $T_{\text {inner }}=10^{7} \mathrm{~K}$, and $\gamma_{\mathrm{o}}$ is the adiabatic index, fixed at $\gamma_{\mathrm{o}}=5 / 3$. The emitted intensity is then simply the Planck function evaluated at this local temperature. The local velocity of the emitting gas is provided by the GR hydrodynamical simulations, which allows us to compute the redshift factor and translate the emitted intensity to the intensity as observed by the distant static observer.

As we are only interested in studying the impact of the disc, or more precisely the parameter of the unstable zone, we look at a black hole with an inclination with respect to the observer of $70^{\circ}$; as more than half of the HFQPO sources have a high inclination, this is not a strong restriction. Changing the inclination causes a change in the strength ratio between peaks but not in their distribution. On the right of Fig. 1 we show a typical disc image for an inclination of $70^{\circ}$ for the case of $a=0.5$ and the RWI occurring at $r_{\mathrm{c}}=8.5 r_{g}$.

\section{What causes the power-density spectrum mode selection?}

As mentioned in Sect. 2, one of the interesting features of the RWI to explain HFQPOs is the fact that the strongest dominant mode is not always the $m=1$ and that multiple higher modes can occur (Tagger \& Varnière 2006); this was studied in semianalytical form only however. Here, we use NOVAs to numerically explore the different PDSs that we can obtain from the RWI while taking into account all physical effects induced by the gravity of the black hole.

This is of particular interest as several integer ratios between the frequency of the observed HFQPOs have been detected, sometimes in the same objects ${ }^{5}$. It is therefore interesting to study the changes required for the RWI to show such different ratios in its PDS and see if those are compatible with observations.

\subsection{Synthetic observations of the RWI exhibiting 3:2 and $3: 4$ ratios}

From analytical and semi-analytical work (see for example Lovelace \& Romanova 2014) we know that the local conditions where the RWI grows are what trigger the growth of one mode over another and in turn the strengths of each mode directly impacts the PDS. In the following section we focus on only one spin $(a=0.995)$ and position $\left(r_{\mathrm{c}}=2.7 r_{g}\right)$ where the RWI is

\footnotetext{
5 It is important to note that, most of the time, these are not detected in individual observations but while accumulating several observations together with different criteria. See for example Miller et al. (2001), Remillard et al. (2002), Varnière \& Rodriguez (2018) for the occurrence of the 3:2 and 3:4 ratios in XTE J1550-564.
}

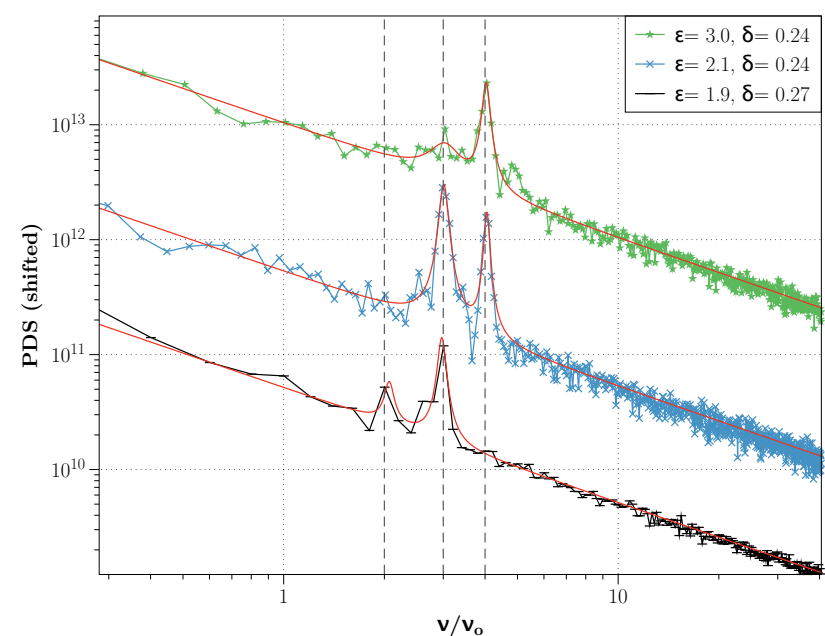

Fig. 2. Three examples of resulting PDS for the same spin $(a=0.995)$ and position $\left(r_{\mathrm{c}}=1.35 r_{\mathrm{S}}\right)$ but with different parameters of the system. For visibility the PDS are vertically shifted.

triggered but look at different local conditions (see Eq. 3 for the parametrization), meaning the (unstable) zone where the RWI is active will be different. We can then see how the local condition affect the PDS, especially focusing on which modes are selected and how they relate to one another when present. We saw in previous studies of the RWI (Tagger \& Varnière 2006; Vincent et al. 2013; Casse et al. 2017; Casse \& Varniere 2018) that every simulation triggers a lot of modes, with up to $m=19$ being detectable in Fourier space; though lower modes tend to be a lot stronger, especially once the instability reaches saturation. Because of the timescale near the last stable orbit of a blackhole of ten solar masses, we mostly observe the saturated state of the instability in a standard observation. We have therefore first performed several simulations with the same aforementioned corotation radius $r_{\mathrm{c}}=2.7 r_{g}$ but with various $\varepsilon$ and $\delta$ parameters leading to different geometries of the unstable zone. A typical GRHD simulation example and the related ray-tracing synthetic observation can be found in Fig. 1. Once a simulation has reached the saturation stage, namely when the amplitude of the fluctuations remains approximately constant, we stop the simulation and proceed to use GYOTO in order to compute the light curve of the simulation as perceived by a remote observer. We stress here that the combination of the GR-AMRVAC code and the GYOTO code encompasses all general relativistic effects at work upon both the disc and its radiative emission, here providing reliable synthetic observations.

Using the combination of GR-AMRVAC and GYOTO we obtain the light curve and then the PDS where the different peaks of HFQPOs can be detected.

When computing those PDSs from the simulations, we often get only one dominant peak or with a small, that is, close to the detection level, secondary peak, as we can see on the upper curve of Fig. 2. In a few cases ${ }^{6}$ we see that multiple peaks are well above the detection level. Figure 2 shows examples of the most commonly observed integer ratios in black-hole binaries, namely the 2:3 and 3:4 ratios, both of those having been observed in GRO J1655-40 (Strohmayer 2001) and in XTE J1550-564 (Varnière \& Rodriguez 2018), respectively.

6 We did not run enough simulations to statically determine the rarity of one case compared to another. 
It is interesting to note that for XTE J1550-564 a case of 2:3 has also been detected by Remillard et al. (2002) by adding together observations with similar low-frequency QPO types ${ }^{7}$ making it the first source where multiple integer ratios have been detected. Not only does Fig. 2 prove the ability of the RWI to create a detectable modulation of the flux, but it also demonstrates that several integer ratios of the peak distribution can be observed in the PDS of the same object, depending on the local conditions in the inner region where the RWI is active.

As we have not performed simulations for a wide range of parameters, we cannot be sure of the minimal level of change required in the system to get different peak ratios. Nevertheless, in the framework of the three RWI simulations presented here, such differences in the integer ratio of the peak distribution are associated with a relatively large change of the parameters of the unstable zone of about $30 \%$. This means that a measured change of $30 \%$ in the parameters of the inner region is enough to trigger a change from a ratio of 2:3 to one of 3:4 in HFQPOs.

\subsection{How much change is seen in the observations?}

The physical variations in the inner region of the system required by the RWI in order to be able to explain a change in peak ratio are strong enough to be not straightforward. For that reason, we decided to look at the observations of the source XTE J1550-564 which has the highest number of different detected HFQPOs to date, along with several distinct integer ratio detections. Taking into account the 1998-1999 and 2000 outbursts, we have about 30 observations with an identified HFQPO which is enough to see if the needed variation of about $30 \%$ of the parameters of the inner region of the disc is compatible with the data.

Using RXTE, other than the QPO itself, we rarely have a direct link to the parameters of the unstable zone in the disc ${ }^{8}$. Instead we focus on the spectral parameters associated with each observation; indeed the RWI is expected to arise near the last stable orbit and therefore impact the temperature in the inner region of the disc as well as the comptonized component. Using data from Sobczak et al. (2000), Remillard et al. (2002), we plot in Fig. 3 the photon index $\Gamma$ versus the temperature at the inner edge of the disc for all the observations of XTE J1550-564 where a HFQPO was detected. We see that the temperature at the inner edge of the disc varies by about a factor of two while the photon index varies by close to $30 \%$.

While those changes in the spectral parameters do not imply a similar change in the inner region where the RWI develops, this region will have to "respond" to those changes in the overall disc and corona. Faced with a change in temperature at the inner edge of the disc of about $100 \%$, it is conceivable to have the unstable region, which is not very far from the inner edge of the disc, change by the factor of $30 \%$ needed for the RWI to explain a wide variety of modes. As a result one can safely assume that a variation of typically $30 \%$ in the parameters of the inner disc cannot be ruled out by actual data from XTE J1550-564. At the same time these observations explain why it is rare to see multiple ratios in one system. Under such circumstances, RWI naturally provides an explanation for the HFQPOs frequency ratio shift within the same astrophysical system.

\footnotetext{
7 How using the LFQPO characteristics to uncover the behaviour of the HFQPO relates to the instability presented here has been shown in more detail in Varnière et al. (2012).

8 See Varnière et al. (2016) for more details on how we can get access to these parameters.
}

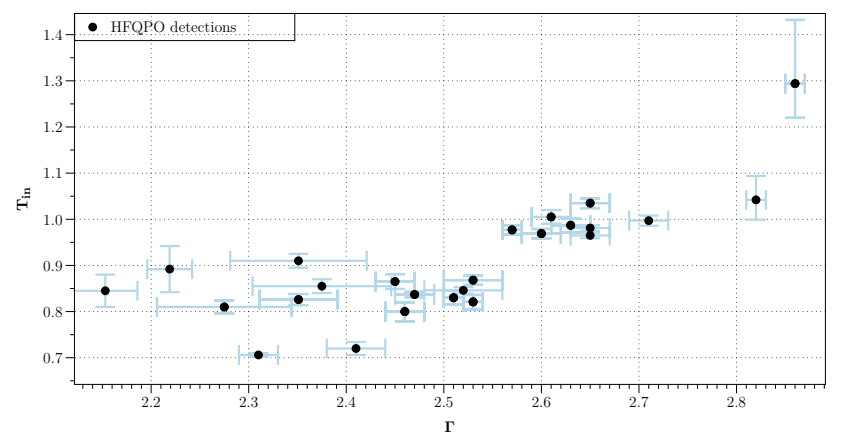

Fig. 3. Photon index $\Gamma$ vs. the temperature at the inner edge of the disc for all the observations of XTE J1550-564 where a HFQPO was detected. We see that the temperature at the inner edge of the disc varies by about a factor of two while the photon index varies by close to $30 \%$. Data from Sobczak et al. (2000), Remillard et al. (2002).

\section{Impact of the spin on the saturation level of the Rossby wave instability}

Because of their high frequency, HFQPOs are often thought to originate from the inner region of a disc close to its last stable orbit, sometimes even requiring a non-zero spin, as was argued by Strohmayer (2001) for the case of GRO J1655-40. Therefore, there is a link between the frequency of the HFQPO and the spin of the black-hole, but this link is hard to pinpoint observationally. Indeed, while HFQPOs sometimes appear in pairs of integerrelated frequencies making it easier to identify the fundamental frequency, they most often do not and we do not know how the observed peak relates to the fundamental frequency.

Here we are exploring the possibility of another observable which can be impacted more directly by the spin of the black hole around which the RWI develops, namely the maximum rms amplitude a HFQPO can attain.

\subsection{Saturation of the Rossby wave instability as a function of spin}

One of the major conclusions of Casse \& Varniere (2018) is that the saturation level of the RWI depends on the position where the instability occurs but not on the spin of the black hole, meaning that for the same corotation radius (i.e. same observed frequency), the spin of the black hole has only a marginal effect on the maximum saturation the instability can reach. In this section we use simulations that appeared in Casse \& Varniere (2018) and compare the saturation levels of different spin/position couples when the RWI develops in the inner region of the disc associated with that spin.

In the left panel of Fig. 4 we show the evolution and saturation of the maximum of the surface density variation under the influence of the RWI (computed by $\left|\sigma-\sigma_{\mathrm{o}}\right|, \sigma_{\mathrm{o}}$ being the equilibrium value of the density) as function of the time normalized by the corotation time for mild to extreme spin. We see that as the spin increases, the saturation level of the instability increases, as well as the time to reach saturation. While the change in time to reach saturation might seem important (reach about 40\%) when taking into account the actual timescale at the corotation, it is an extremely small difference, below what we can currently detect. What is more interesting is the net increase in the saturation level (the plot is in log to see the behaviour from the start of the simulation) by more than a factor of two between mild and extreme spin. The right panel of Fig. 4 summarises the saturation level 

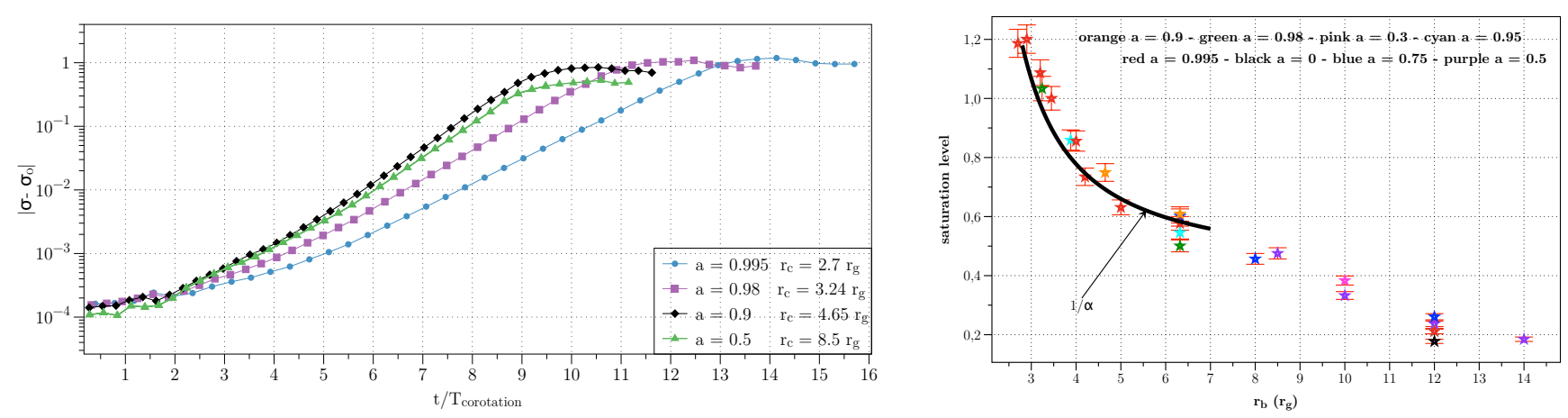

Fig. 4. Left: evolution of the maximum of $\left|\sigma-\sigma_{\mathrm{o}}\right|$ as a function of time normalized by the corotation time for four different spins, showing how an increased spin causes a higher saturation (it is a logscale in y and the saturation varies by more than a factor of two). The cases represented here are, from right to left, blue circle $a=0.995$ at $r_{\mathrm{c}}=2.7 r_{g}$, purple square $a=0.98$ and $r_{\mathrm{c}}=3.24 r_{g}$, black diamond $a=0.9$ with $r_{\mathrm{c}}=4.65 r_{g}$ and green triangle $a=0.5$ at $r_{\mathrm{c}}=8.5 r_{g}$. Right: saturation levels reached by RWI in our simulation sample with corotation radius and spin parameter ranging from $r_{\mathrm{c}}=14 r_{g}$ to $r_{\mathrm{c}}=2.7 r_{g}$ and from $a=0$ to $a=0.995$. All simulations have the same set of parameters regarding the unstable zone, namely the same $\varepsilon$ and $\delta$. It clearly appears that the saturation level increases as the corotation radius is decreasing meaning that higher spin will lead to the RWI occurring nearer to the black hole, which then leads to enhanced saturation levels in the instability.

obtained from all simulations performed with the same geometry of the unstable region, namely with the exact same values of $\varepsilon$ and $\delta$. In this figure one can easily see that the saturation level of the RWI is increasing as the corotation radius decreases. For $r_{\mathrm{c}}>4 r_{g}$ the increase follows the variation of the local density in the disc while for the closest corotation radii $\left(r_{\mathrm{c}}<4 r_{q}\right)$ the increase depends on general relativistic effects and is inversely proportional to the lapse. Having the inner part of an accretion disc so close to the black hole is only possible for high spin values so one prediction of the RWI model is that, for a similar setup, a higher spin, that is, a smaller inner radius assuming the disc reaches its last stable orbit, will mean a higher saturation level.

This is not something we can straightforwardly compare with observations. Indeed, in Fig. 4 the physical conditions in the system, or more precisely in the region where the RWI is active, are similar ${ }^{9}$ while we have no simple way to constrain the amplitude of the differences between the inner disc conditions of two observations of two different objects.

\subsection{Root mean square amplitude of HFQPOs as a function of spin}

In order to assess a potential relationship between rms amplitude and the spin of the black hole, we look at the overall behaviour of the rms of HFQPOs in all the objects for which the spin is known. By taking into account the overall envelope shape of the rms versus spin we get the evolution of the "maximum" (to this day) cases for all the objects. If we assume that this maximum is reached for each object in similar conditions in terms of disc properties, then using Fig. 4 the RWI predicts the maximum achievable rms to increase with the spin of the black hole. This is something we can check with the limited sample of spin estimates we have for the HFQPO system.

\subsubsection{Spin determination in observations}

As we are using only published values and did not redo any of the data reductions, there exists only a limited sample of sources for which both spin measurements, calculated using any method,

\footnotetext{
9 One can see how close the disc parameters are in Fig. 1 of Casse \& Varniere (2018).
}

Table 1. Values of the spins for the five HFQPO sources that have a spin estimate either from the continuum fitting and the inferred position of the last stable orbit (CF) or the iron line and reflection fitting model (FeK) or both.

\begin{tabular}{cccc}
\hline \hline Source & CF & FeK & Used \\
\hline 4U1630-472 & & $>0.95$ & $>0.95$ \\
H1743-322 & $0.2 \pm 0.3$ & & $0.2 \pm 0.3$ \\
XTE J1550-564 & $0.34 \pm 0.28$ & $0.55 \pm 0.1$ & $0.36 \pm 0.29$ \\
GRO J1655-40 & $0.7 \pm 0.1$ & $>0.9$ & $0.8 \pm 0.2$ \\
XTE J1650-500 & & $0.84-0.98 / \sim 0.998$ & $0.84-0.998$ \\
& & $\geq 0.93 / 0.977_{-0.07}^{+0.06}$ \\
\hline
\end{tabular}

Notes. The last column is the range displayed in Fig. 5; when several values exist, we choose a range that encompasses all values.

and an observed HFQPO (with published rms value) have been obtained. Here we use spin estimates from one (or both) of the two main methods, namely the continuum fitting (CF) method and the iron line fitting (FeK) method. Both methods have been shown to have limitations. Indeed, in order to get the spin from either fit we need to have a good grasp on the disc fitting model (Kolehmainen et al. 2011). Keeping in mind the known limitations, we can still use the limited sample of published estimates to compare with this prediction of the RWI to see if there is an overall agreement or contradiction.

Our sample is composed of 4U1630-472 (red) with data from King et al. (2014), Klein-Wolt et al. (2004), H1743-322 (blue) with data from Steiner et al. (2012), Homan et al. (2003), XTE J1550-564 (green) with data from Steiner et al. (2011), Remillard et al. (2002), GRO J1655-40 (purple) with data from Shafee et al. (2006), Reis et al. (2009), Strohmayer (2001) and XTE J1650-500 (black) with data from Miller et al. (2002), Miniutti et al. (2004), Walton et al. (2012), Reis et al. (2013), Homan et al. (2003).

As we can see from Table 1, in our sample we have two objects that have only one determination, two objects that have spin estimates from both methods, and one object that has been measured several times with the same method but by different groups on different observations.

We see good agreement of both methods for XTE J1550-564 and we see that three of the four iron-line-fitting spin estimations of XTE J1650-500 are coherent with one another, while 


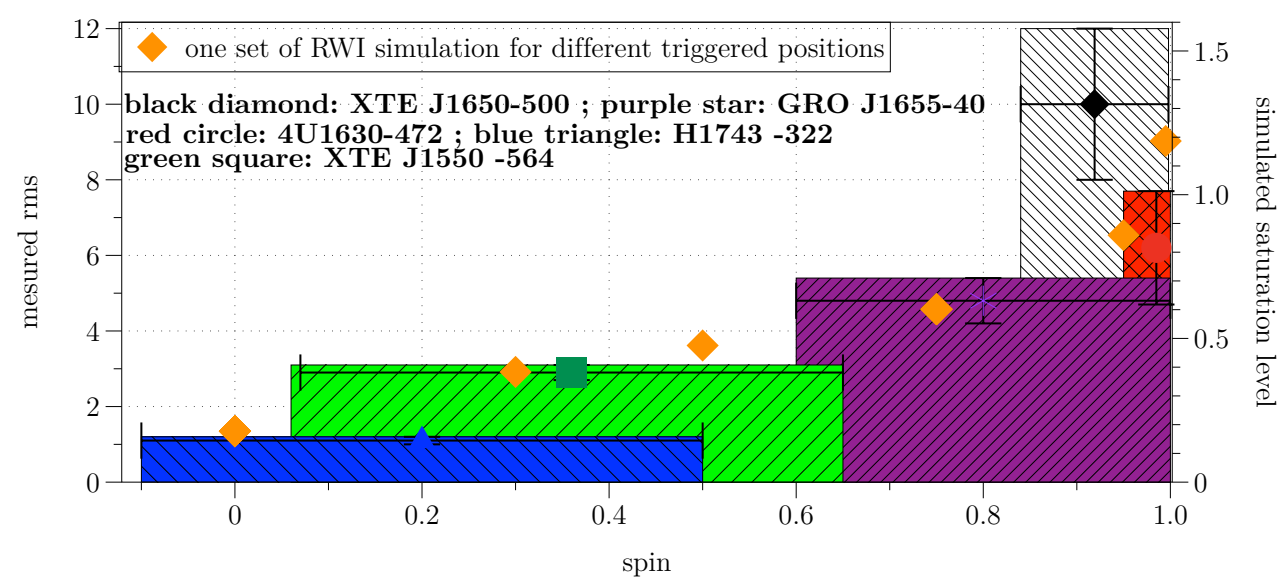

Fig. 5. Evolution of the rms amplitude of published HFQPOs as function of spin, showing a behaviour compatible with higher spin source tending to have higher maximum rms. See the text for references. For comparison we added the value of the simulation setup from Fig. 4. as orange diamond. We see that, within the error bar, the observed data seems to follow a similar evolution as function of spin as the one predicted by the RWI. the fourth estimate is fully compatible with only one of the four and at the same time being slightly outside the error bars of the last two. The only real disagreement we have between the two methods is for GRO J1655-40 where the variability (HFQPOs) and the inferred position of the last stable orbit method provides a spin of $0.7 \pm 0.1$ (Strohmayer 2001) while the use of the disc reflection signatures requires the spin to be higher than 0.9 (Reis et al. 2009).

Because our sample is so small with only two objects having spin agreement with both methods, we need to take into account all of the known objects with a spin assessment made with one of the methods. Using all the data from McClintock et al. (2014) and Reynolds (2014) we obtain a slightly bigger sample of seven objects with spin estimates from at least one of the two methods. Of those, four have coherent spin estimates from various studies using the same method with different observations. One of the systems showing a spin estimate discrepancy, namely $4 \mathrm{U}$ 1543-47, was studied by Morningstar \& Miller (2014) using both techniques simultaneously. These latter authors found that the difference between the two methods came mostly from the hypothesis used, for example the disc inclination coming from the iron line fitting and the inclination used in the continuum fitting were not the same. Morningstar \& Miller (2014) showed that using the same inclination led them to obtain coherent spin measurements with both methods. The value of the spin they found with this method was consistent with both other values within errors. Therefore, we now have five out of seven systems with reliable spin values, which gives some credibility to the method.

It is worth considering that GRO J1655-40, the case with mismatch spins in our sample, is thought to perhaps have spin misalignment. As shown in Morningstar \& Miller (2014) this could lead to the difference in the spin estimation and a more thorough estimate of the spin is needed here taking into account both methods.

As a result we decided to use a large range of spin values by expanding the error bars in order to encompass all the available spin estimates. These are shown in the last column of Table 1.

\subsubsection{Evolution of the maximum rms as a function of spin}

With the rms published in the aforementioned publications (Sect. 5.2.1) and the spin estimate from Table 1, Fig. 5 shows how the maximum rms evolves as a function of the spin. We choose to represent the distribution of rms versus spin as a column, visually expressing the error bars with coloured boxes.
This is a sparse sampling with large error bars, but it shows agreement with the predicted behaviour of a RWI-based modulation, that is, the maximum rms amplitude will increase as a function of spin. For reference, in Fig. 5 we also added the results from the set of RWI simulations shown in Fig. 4, shown here with orange diamond symbols. These are added to show the trend but do not represent a "fit" of the data as we do not know enough about the conditions of the system at the time of the observation to run the adapted simulations.

For the moment we have only very few sources with known spin and few sources with known HFQPOs; the intersection, where both spin and HFQPOs are known, is the five sources presented here. It would be interesting to continue this study by checking all the sources with known spins for HFQPOs and also to measure the spin of sources known to harbour HFQPOs. This could almost double the number of points (we have eight known sources with HFQPOs detected as of now) which would lend more credence to the behaviour presented here.

\section{Conclusions}

Thanks to NOVAs, our newly developed GR fluid code, and the associated general relativistic ray-tracing, we have created synthetic observations of a Kerr black-hole system with a RWI developing in the disc. This allowed us, for the first time, to study observables from the RWI more directly. Here we present two predictions arising from associating the RWI with the cause for HFQPOs.

The first prediction, which is now observed, concerns the ability of one system to display several integer ratio frequencies in its power distribution spectra at different times. As this became observed, we turned to explore what is required in order to achieve this change. We showed that the RWI was able to reproduce the PDS with different dominant modes and integer ratios between them if the inner region of the system changed by about $30 \%$. Using published data, we showed that such changes are compatible with observations. Using more precise observations, such as NICER data, will help in constraining the conditions required to detect certain frequencies and ratios. Indeed, more simulations, linked with higher-resolution observations, are needed in order to pinpoint the main component behind the mode selection of HFQPOs.

The second prediction pertains to the link between the maximally attainable rms and the spin of the black hole. Indeed, under the same setup, the RWI saturation was seen to increase with the spin of the black-hole. This is more tricky as spin estimation 
is not something the entire community agrees on. Nevertheless, we could not pass up the opportunity to use the limited sample of published estimates to compare with this prediction of the RWI. Using published data we find that astrophysical systems in our sample exhibit maximal rms levels that are compatible with the RWI prediction, namely that the maximal rms level increases with the spin of the black hole. We aim to follow this up by looking for more HFQPOs in sources with known spins, especially in the new NICER data.

Acknowledgements. We thanks the anonymous referee for his comments, which helped to improve this paper. PV thanks J. Steiner for his advice concerning the spin determinations in black-hole binaries. We acknowledge the financial support of the UnivEarthS Labex program at Sorbonne Paris Cité (ANR-10LABX-0023 and ANR-11-IDEX-0005-02).

\section{References}

Altamirano, D., \& Belloni, T. 2012, ApJ, 747, L4

Belloni, T. M., \& Altamirano, D. 2013, MNRAS, 432, 19

Belloni, T., Méndez, M., \& Sánchez-Fernández, C. 2001, A\&A, 372, 551

Belloni, T. M., Sanna, A., \& Méndez, M. 2012, MNRAS, 426, 1701

Casse, F., \& Varniere, P. 2018, MNRAS, 481, 2736

Casse, F., Varniere, P., \& Meliani, Z. 2017, MNRAS, 464, 3704

Homan, J., Klein-Wolt, M., Rossi, S., et al. 2003, ApJ, 586, 1262

Kerr, R. P. 1963, Phys. Rev. Lett., 11, 237

King, A. L., Walton, D. J., Miller, J. M., et al. 2014, ApJ, 784, L2

Klein-Wolt, M., Homan, J., \& van der Klis, M. 2004, Nucl. Phys. B Proc. Suppl., 132,381

Kolehmainen, M., Done, C., \& Díaz Trigo, M. 2011, MNRAS, 416, 311

Lin, M.-K. 2012, MNRAS, 426, 3211

Lovelace, R. V. E., \& Hohlfeld, R. G. 1978, ApJ, 221, 51

Lovelace, R. V. E., \& Romanova, M. M. 2014, Fluid Dyn. Res., 46, 041401
Lyra, W., \& Mac Low, M.-M. 2012, ApJ, 756, 62

McClintock, J. E., Narayan, R., \& Steiner, J. F. 2014, Space Sci. Rev., 183, 295 Meheut, H., Casse, F., Varniere, P., \& Tagger, M. 2010, A\&A, 516, A31 Meheut, H., Keppens, R., Casse, F., \& Benz, W. 2012, A\&A, 542, A9

Mignone, A., \& McKinney, J. C. 2007, MNRAS, 378, 1118

Miller, J. M., Wijnands, R., Homan, J., et al. 2001, ApJ, 563, 928

Miller, J. M., Fabian, A. C., Wijnands, R., et al. 2002, ApJ, 570, L69

Miniutti, G., Fabian, A. C., \& Miller, J. M. 2004, MNRAS, 351, 466

Morningstar, W. R., \& Miller, J. M. 2014, ApJ, 793, L33

Reis, R. C., Fabian, A. C., Ross, R. R., \& Miller, J. M. 2009, MNRAS, 395, 1257

Reis, R. C., Miller, J. M., Reynolds, M. T., et al. 2013, ApJ, 763, 48

Remillard, R. A., \& McClintock, J. E. 2006, ARA\&A, 44, 49

Remillard, R. A., Muno, M. P., McClintock, J. E., \& Orosz, J. A. 2002, ApJ, 580, 1030

Reynolds, C. S. 2014, Space Sci. Rev., 183, 277

Shafee, R., McClintock, J. E., Narayan, R., et al. 2006, ApJ, 636, L113

Sobczak, G. J., McClintock, J. E., Remillard, R. A., et al. 2000, ApJ, 544, 993

Steiner, J. F., Reis, R. C., McClintock, J. E., et al. 2011, MNRAS, 416, 941

Steiner, J. F., McClintock, J. E., \& Reid, M. J. 2012, ApJ, 745, L7

Strohmayer, T. E. 2001, ApJ, 552, L49

Tagger, M., \& Melia, F. 2006, ApJ, 636, L33

Tagger, M., \& Varnière, P. 2006, ApJ, 652, 1457

Varnière, P., \& Rodriguez, J. 2018, ApJ, 865, 113

Varnière, P., \& Tagger, M. 2006, A\&A, 446, L13

Varnière, P., Tagger, M., \& Rodriguez, J. 2011, A\&A, 525, A87

Varnière, P., Tagger, M., \& Rodriguez, J. 2012, A\&A, 545, A40

Varnière, P., Mignon-Risse, R., \& Rodriguez, J. 2016, A\&A, 586, L6

Varnière, P., Casse, F., \& Vincent, F. H. 2018, in Proceedings of the XMMNewton 2018 Science Workshop, Time-domain Astronomy: A High Energy View Esac, Madrid, Spain, 13-15 June 2018

Vincent, F. H., Paumard, T., Gourgoulhon, E., \& Perrin, G. 2011, Classical Quantum Gravity, 28, 225011

Vincent, F. H., Meheut, H., Varniere, P., \& Paumard, T. 2013, A\&A, 551, A54

Vincent, F. H., Paumard, T., Perrin, G., et al. 2014, MNRAS, 441, 3477

Walton, D. J., Reis, R. C., Cackett, E. M., Fabian, A. C., \& Miller, J. M. 2012, MNRAS, 422, 2510 